\title{
GTC/CanariCam mid-IR imaging of the fullerene-rich planetary nebula IC 418
}

\author{
J. J. Díaz-Luis ${ }^{1,2}$, D. A. García-Hernández ${ }^{1,2}$, A. Manchado ${ }^{1,2,3}$, \\ P. García-Lario ${ }^{4}$, E. Villaver ${ }^{5}$ and G. García-Segura ${ }^{6}$ \\ ${ }^{1}$ Instituto de Astrofísica de Canarias, C/ Vía Láctea s/n, E-38205 La Laguna, Spain \\ email: jdiaz@iac.es \\ ${ }^{2}$ Departamento de Astrofísica, Universidad de La Laguna (ULL), E-38206 La Laguna, Spain \\ ${ }^{3}$ Consejo Superior de Investigaciones Científicas, Madrid, Spain \\ ${ }^{4}$ Herschel Science Centre, ESAC-ESA, E-28691 Villanueva de la Cañada, Madrid, Spain \\ ${ }^{5}$ UAM, Departamento de Física Teórica C-XI, E-28049 Madrid, Spain \\ ${ }^{6}$ Instituto de Astronomía, UNAM, 22860 Ensenada, México
}

\begin{abstract}
The formation process(es) of fullerenes in space is still uncertain and several mechanisms have been proposed in the literature. In particular, the most accepted idea to explain the simultaneous presence of fullerenes and PAH-like emission in the H-rich circumstellar envelopes of $\mathrm{PNe}$ is that these molecular species may be formed from the photochemical processing of a carbonaceous compound with a mixture of aromatic and aliphatic structures, which should be a major constituent of their circumstellar envelopes. Here we present seeing-limited narrow-band mid-IR GTC/CanariCam images of the fullerene-containing PN IC 418. The narrow-band images cover the $9-13,11.3$, and $17.4 \mu \mathrm{m}$ emission features (and their adjacent continua) in this extended PN. We study the relative sub-arcsecond spatial distribution of the nebula in these filters with the intention of getting some clues about the formation process of fullerenes in H-rich circumstellar environments.
\end{abstract}

Keywords. Astrochemistry, molecular processes, stars: evolution, planetary nebulae: individual (IC 418), AGB and post-AGB, dust.

\section{Introduction}

The detection of fullerenes around old stars together with the recent identification of $\mathrm{C}_{60}{ }^{+}$as a diffuse interstellar band (DIB) carrier (the only DIB carrier known to date; Campbell et al. 2015) indicate that fullerenic molecular nanostructures should be ubiquitous in the interstellar medium (ISM), playing an important role in many aspects of circumstellar/interstellar Chemistry and Physics.

The formation process of fullerenes in space is still uncertain and several mechanisms have been proposed in the literature. The most suitable ones being: i) the formation in H-poor environments (Goeres \& Sedlmayr 1992); ii) high-temperature formation in C-rich environments (Jäger et al. 2009); iii) photochemical processing of hydrogenated amorphous carbon grains (HACs; García-Hernández et al. 2010); and iv) photochemical processing of large PAHs (Berné \& Tielens 2012).

At present, the most likely explanation for the simultaneous presence of fullerenes and PAH-like emission in the H-containing circumstellar environments of planetary nebulae $(\mathrm{PNe})$ is that they may be formed by the decomposition of a carbonaceous material with a mixture of aromatic and aliphatic structures; e.g., similar to hydrogenated amorphous carbon grains (HAC-like hereafter; García-Hernández et al. 2010; Micelotta et al. 2012). Such carbon compounds should be a major constituent of the circumstellar envelope of 

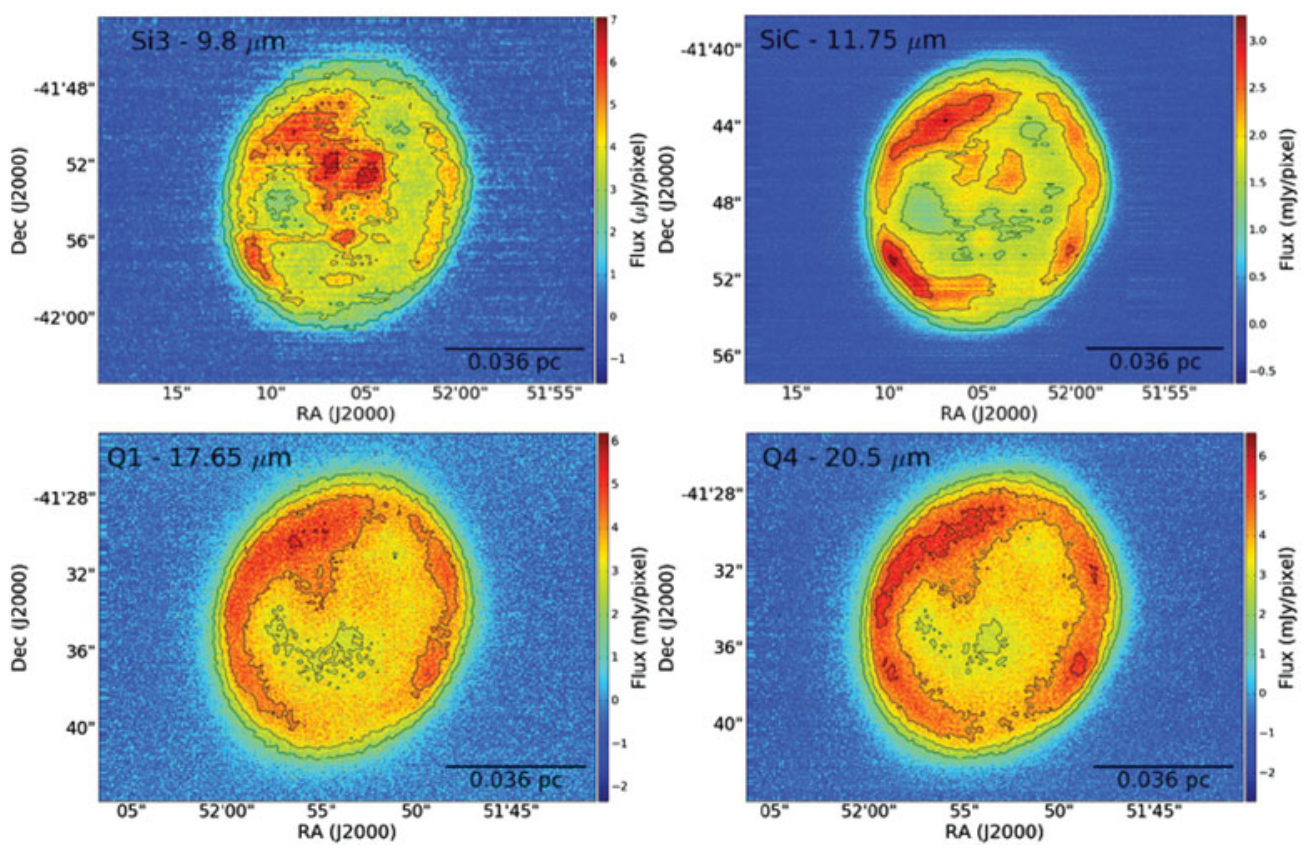

Figure 1. Flux-calibrated GTC/CanariCam mid-IR images of the $\mathrm{C}_{60}-\mathrm{PN}$ IC 418 in the Si3, $\mathrm{SiC}, \mathrm{Q} 1$, and Q4 filters.

C-rich evolved stars. This fullerene formation scenario (the HAC scenario) is suggested by the coexistence of a complex mix of aliphatic and aromatic species such as HACs, $\mathrm{PAH}$ clusters, fullerenes, and small dehydrogenated carbon clusters (possibly planar $\mathrm{C}_{24}$ or a small fragment of a graphene sheet) in PNe of the Magellanic Clouds and our own Galaxy (García-Hernández et al. 2011, 2012).

Mid-IR imaging observations of the different molecular/dust species in PNe with fullerenes may help to understand the formation process of fullerenes in these objects.

\section{GTC/Canaricam mid-IR observations}

Seeing-limited (FWHM $\sim 0.5 "$ ) mid-IR images of the extended fullerene-rich PN IC 418 were obtained with GTC/Canaricam in January and December 2013 by using narrowband filters centered at $11.30 \mu \mathrm{m}$ (PAH2, $\Delta \lambda=0.6 \mu \mathrm{m}), 11.75 \mu \mathrm{m}(\mathrm{SiC}, \Delta \lambda=$ $2.5 \mu \mathrm{m})$, and $17.65 \mu \mathrm{m}(\mathrm{Q} 1, \Delta \lambda=0.9 \mu \mathrm{m})$, as well as in their adjacent continua at $9.8 \mu \mathrm{m}(\mathrm{Si} 3, \Delta \lambda=1.0 \mu \mathrm{m})$ and $20.50 \mu \mathrm{m}(\mathrm{Q} 4, \Delta \lambda=1.0 \mu \mathrm{m})$. The signal-to-noise ratio in the final combined images is $\sim 5-16$. Figure 1 shows the final flux-calibrated mid-IR GTC/Canaricam images of IC 418.

The PAH2 and SiC filters cover the PAH-like feature at $11.3 \mu \mathrm{m}$ and the broad 9-13 $\mu \mathrm{m}$ band (possibly related with HAC-like dust grains; e.g., García-Hernández et al. 2010, 2011), while the Q1 filter covers the $\mathrm{C}_{60}$ emission feature at $17.4 \mu \mathrm{m}$. The Si3 and Q4 filters cover the dust continuum emission at 9.8 and $20.5 \mu \mathrm{m}$, respectively.

\section{Results}

The Si3 image displays the maximum emission near the IC 418 central star, while the $\mathrm{SiC}, \mathrm{Q} 1$, and Q4 images show a ring-like structure (Fig. 1). The similar spatial 

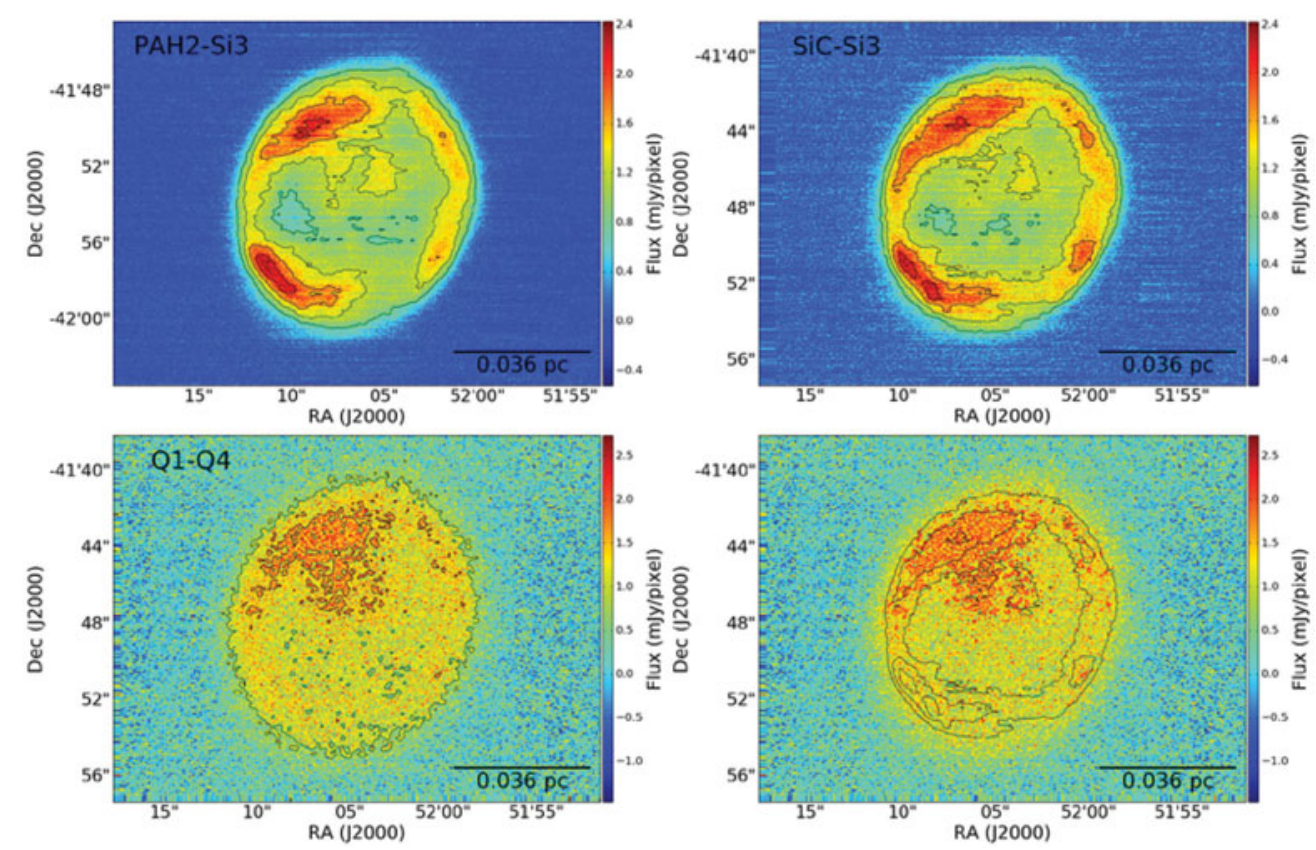

Figure 2. Continuum-subtracted GTC/Canaricam mid-IR images of IC 418: PAH2-Si3, $\mathrm{SiC}-\mathrm{Si} 3$, and Q1-Q4. The bottom-right panel displays the Q1-Q4 continuum-subtracted image $\left(\mathrm{C}_{60}\right.$ emission) with overlaid contours of the $9-13 \mu \mathrm{m}+11.3 \mu \mathrm{m}$ PAH-like (SiC-Si3) emission.

distribution seen in the Q1 and Q4 filters suggests that fullerenes may be co-spatial with the dust continuum emission (e.g., they could be attached to the dust grains) or that both images are dominated by the dust continuum emission (e.g., because of the low feature-to-continuum ratio). Also, the similar ring-like structure seen in the $\mathrm{SiC}$ image (which is dominated by the broad $9-13 \mu \mathrm{m}$ feature) indicates that the $9-13 \mu \mathrm{m}$ band carrier is co-spatial with the dust grains emitting at $20.5 \mu \mathrm{m}$ (Q4). On the other hand, by comparing the Si3 image with public Hubble Space Telescope optical nebular emission images of IC 418, we find that the dust continuum emission at $9.8 \mu \mathrm{m}$ is very similar to the $[\mathrm{O}$ III] nebular emission, indicating that dust grains coexist with ionized material.

In order to study the relative spatial distribution of the $\mathrm{C}_{60}$ molecules, the PAH-like molecules, and the $9-13 \mu \mathrm{m}$ band carrier, we have used the Si3 and Q4 images to subtract the dust continuum contribution. Figure 2 shows the resulting PAH2-Si3, SiC-Si3, and Q1-Q4 continuum-subtracted images as well as a comparison of the spatial distribution of the $\mathrm{C}_{60}(\mathrm{Q} 1-\mathrm{Q} 4)$ and $9-13 \mu \mathrm{m}+11.3 \mu \mathrm{m}$ PAH-like (SiC-Si3) emissions.

The PAH2 and SiC images cover the weak $11.3 \mu \mathrm{m}$ and the broad $9-13 \mu \mathrm{m}$ features (the PAH+HAC-like emission) with different filter widths; moreover, the PAH2-Si3 and $\mathrm{SiC}-\mathrm{Si} 3$ continuum-subtracted images display, as expected because of the weak $11.3 \mu \mathrm{m}$ PAH-like feature, an almost identical spatial distribution. The $9-13 \mu \mathrm{m}$ emission is mainly located in the outer regions of the nebula, defining a ring-like structure with the stronger emission peaks at the northeast and southeast regions. The continuumsubtracted fullerene emission (the Q1-Q4 image in Fig. 2), however, is very weak and mainly located at the northeast, extending from the inner (even near the central star) to the outer regions of the nebula. The weak fullerene emission is quite similar to that of the dust continuum emission at $9.8 \mu \mathrm{m}$, which also coincides with the spatial distribution of the [O III] nebular emission (see above). At the northeast outer regions of the nebula, 

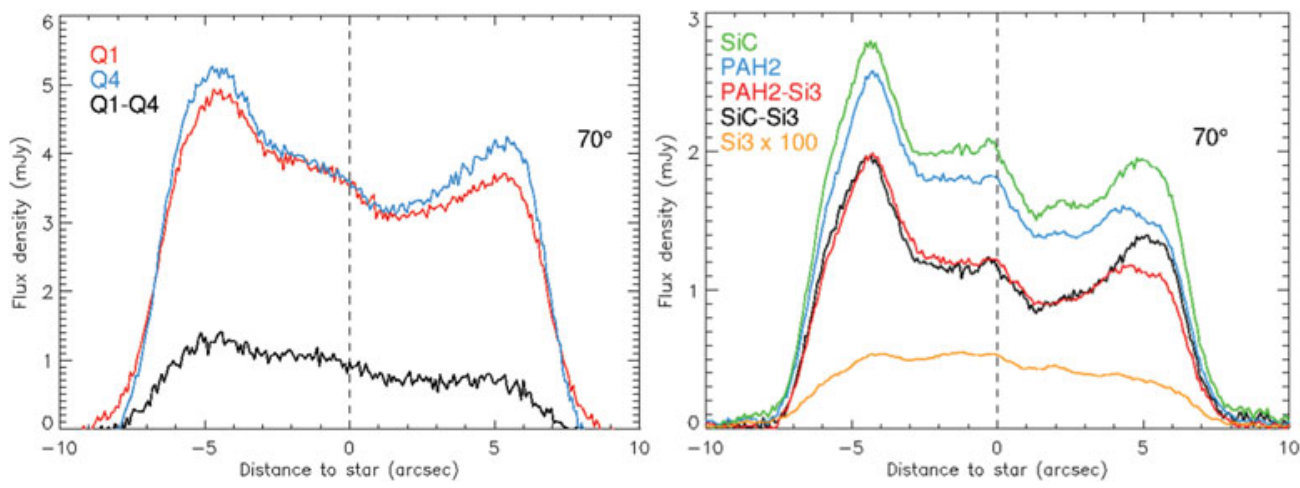

Figure 3. Intensity profiles along the IC 418 nebula at $70^{\circ}$ with respect to the equatorial plane as a function of the distance to the central star. Left panel: intensity profiles in the Q1 $\left(\mathrm{C}_{60}+\right.$ continuum at $\left.17.65 \mu \mathrm{m}\right), \mathrm{Q} 4$ (continuum at $\left.20.5 \mu \mathrm{m}\right)$, and continuum-subtracted Q1-Q4 $\left(\mathrm{C}_{60}\right)$ images. Right panel: intensity profiles in the $\mathrm{Si} 3$ (continuum at $9.8 \mu \mathrm{m}$ ), $\mathrm{SiC}$ and PAH2 (both containing 9-13 $\mu \mathrm{m}$ carrier $+11.3 \mu \mathrm{m}$ PAH-like emission + continuum), and continuum-subtracted $\mathrm{SiC}-\mathrm{Si} 3$ and $\mathrm{PAH} 2-\mathrm{Si} 3$ (9-13 $\mu \mathrm{m}$ carrier $+11.3 \mu \mathrm{m}$ PAH-like emission) images.

fullerenes seem to coexist with the $9-13 \mu \mathrm{m}$ carrier, while they are co-spatial with the dust grains and ionized material in the inner regions closer to the central star.

Figure 3 shows intensity profiles along the IC 418 nebula at $70^{\circ}$ with respect to the equatorial plane as a function of the distance to the central star. The $9-13 \mu \mathrm{m}$ emission peaks at $\sim 4-5$ " from the central star (at both northeast and southwest sides of the nebula), while the weaker fullerene emission mainly extends from the central star to $\sim 5$ " at the northeast of the nebula $(\sim 6300 \mathrm{au})$.

The interpretation of the present images regarding the dominant formation process of fullerenes in PNe is somewhat contradictory. The presence of fullerene emission near the central star, where the $9-13 \mu \mathrm{m}$ emission is much weaker, would suggest that $\mathrm{C}_{60}$ may be a photo-product of the 9-13 $\mu \mathrm{m}$ band carrier. However, fullerenes seem to coexist with the 9-13 $\mu \mathrm{m}$ band carrier at the northeast outer regions of the nebula; something that could indicate that fullerenes may be attached to dust grains or well mixed with the 9-13 $\mu \mathrm{m}$ band carrier. Another possible interpretation may be that fullerenes are not directly linked to the 9-13 $\mu \mathrm{m}$ band carrier. We conclude that higher sensitivity mid-IR images as well as spatially resolved mid-IR spectra along the nebula would be desirable in order to reach a definite answer.

\section{References}

Berné, O. \& Tielens, A. G. G.. M. 2012, Proceedings of the National Academy of Science, 109, 401

Campbell, E. K., Holz, M., Gerlich, D., \& Maier, J. P. 2015, Nature, 523, 322

García-Hernández, D. A., Manchado, A., García-Lario, P. et al. 2010, ApJ, 724, L39

García-Hernández, D. A., Iglesias-Groth, S., Acosta-Pulido, J. A. et al. 2011, ApJ, 737, L30

García-Hernández, D. A., Villaver, E., García-Lario, P. et al. 2012, ApJ, 760, 107

Goeres, A. \& Sedlmayr, E. 1992, A\& A, 265, 216

Jäger, C., Huisken, F., Mutschke, H., Jansa, I. L., \& Henning, Th. 2009, ApJ, 696, 706

Micelotta, E. R., Jones, A. P., Cami, J., Peeters, E. et al. 2012, ApJ, 761, 35 


\section{Discussion}

Guzman-Ramirez: How do you subtract the continuum images? Did you take into account the size of the filter and the slope of the continuum?

DiAz-Luis: No, it is a first approximation and now we will have to flux-calibrate our images. With a simple subtraction we can see the different distributions.

HRIVNAK: You see association of fullerenes with HACs+PAH in NE region but not with HACs+PAH in SE. Can you suggest why the difference?

DiAZ-Luis: Maybe the process (formation) takes place in that part of the nebula and we don't see anything in the south. Photochemical processing of HACs has to take place under certain conditions.

SLOAN: What is the basis for identifying the "9 - $13 \mu \mathrm{m}$ emission" as HAC? Sloan et al. (2014) showed that the broad feature $\sim 11.5 \mu \mathrm{m}$ was a combination of $\mathrm{SiC}+\mathrm{PAHs}$, perhaps Class D PAHs in some cases, but still SiC+PAHs.

DiAz-Luis: The $12 \mu \mathrm{m}$ plateau is certainly not $\mathrm{SiC}$. SiC feature has been detected in over 700 carbon stars and the $\mathrm{SiC}$ feature does not look like the $12 \mu \mathrm{m}$ plateau. This is due to superposition of $\mathrm{C}-\mathrm{H}$ out-of plane bending mode of aliphatic side groups. 